\title{
UM FOTÔMETRO MULTI-LED MICROCONTROLADO, PORTÁTIL E DE BAIXO CUSTO
}

\author{
Edvaldo da Nóbrega Gaião, Everaldo Paulo de Medeiros, Wellington da Silva Lyra, Pablo Nogueira Teles Moreira, \\ Pablo Cavalcante de Vasconcelos, Edvan Cirino da Silva* e Mário César Ugulino de Araújo \\ Departamento de Química, Centro de Ciências Exatas e da Natureza, Universidade Federal da Paraíba, CP 5093, \\ 58051-970 João Pessoa - PB
}

Recebido em 21/7/04; aceito em 29/3/05; publicado na web em 24/8/05

\begin{abstract}
A MULTI-LED, MICROCONTROLLED, PORTABLE AND INEXPENSIVE PHOTOMETER. A microcontrolled, portable and inexpensive photometer is described. It uses six light-emitting diodes (LEDs) as radiation sources and a phototransistor as detector, as well as a microcontroller (PIC - Programmable Controller of Interruption). This device provided total autonomy to the proposed photometer, which was successfully applied to determination of $\mathrm{Fe}^{2+}$ in ferrous syrups and of seven clinical biochemical parameters. As the components are cheap ( U $\$ 30.00)$ and easy to find, the proposed photometer is an economical alternative for routine chemical analyses in small laboratories, for research and teaching. Being portable and microcontrolled, it allows doing field chemical analyses.
\end{abstract}

Keywords: Multi-LED photometer; microcontroller; ferrous ion.

\section{INTRODUÇÃO}

Diodos emissores de luz (LEDs) ${ }^{1}$ têm proporcionado muitas vantagens quando utilizados em instrumentação óptica ${ }^{1,2}$, especialmente para espectrofotometria de absorção molecular UV-VIS. De fato, fotômetros simples, portáteis e de baixo custo, que empregam LED como fonte de radiação, têm sido desenvolvidos e usados em diversas aplicações analíticas ${ }^{1-7}$.

Entre os fotômetros descritos na literatura, existem alguns que usam dois ou mais LEDs com máximos de emissão em diferentes comprimentos de onda, o que os tornam potencialmente aplicáveis a análises multicomponentes ${ }^{2,8}$. Contudo, a necessidade do uso de um microcomputador para aquisição e/ou tratamentos dos dados dificulta o uso desses instrumentos em laboratórios com poucos recursos financeiros e/ou em análises químicas de campo. Mesmo o fotômetro multi-LED proposto por Cantrell e colaboradores", que utiliza um microcontrolador ("PICProgrammable Controller of Interruption") ${ }^{10}$ para aquisição automática de sinais, necessita de um microcomputador para tratamento dos dados. Para contornar essa limitação, desenvolveu-se neste trabalho um fotômetro multi-LED baseado em um microcontrolador PIC de outra série, que permite dispensar totalmente o uso de um microcomputador.

Para avaliar o desempenho do fotômetro proposto, realizaramse determinações de íon ferroso em amostras de xarope e de proteínas totais, albumina, glicose, uréia, cálcio, cloreto e hemoglobina em amostras de soro sangüíneo.

\section{PARTE EXPERIMENTAL}

\section{Descrição do fotômetro proposto}

O instrumento proposto emprega somente componentes baratos e de fácil aquisição. Ele foi acondicionado em uma caixa plástica pequena com dimensões $15 \times 10 \times 5 \mathrm{~cm}$. Como fontes de radiação, o fotômetro utiliza seis LEDs com as seguintes caracte-

*e-mail: edvan@quimica.ufpb.br rísticas: violeta $\left(\lambda_{\text {máx }}=430 \mathrm{~nm}\right)$, azul $\left(\lambda_{\text {máx }}=485 \mathrm{~nm}\right)$, verde $\left(\lambda_{\text {máx }}\right.$ $=560 \mathrm{~nm})$, amarelo $\left(\lambda_{\text {máx }}=580 \mathrm{~nm}\right)$, laranja $\left(\lambda_{\text {máx }}=600 \mathrm{~nm}\right) \mathrm{e}$ vermelho $\left(\lambda_{\text {máx }}=660 \mathrm{~nm}\right)$, conforme espectros de emissão mostrados na Figura 1.

Os LEDs foram montados em um suporte circular adaptado ao eixo de uma chave eletro-mecânica de seis posições que permite, ao mesmo tempo, ligar e posicionar o LED desejado em frente ao detector (Figura 2).

O detector escolhido para esse protótipo é um fototransistor, pois é barato e de fácil aquisição e apresenta uma boa relação sinal-ruído. Entre o LED e o detector foi colocado um suporte de alumínio onde se encaixa uma cela de detecção com caminho óptico de $1,0 \mathrm{~cm}$. A cela de detecção pode ser do tipo convencional ou em fluxo (Figura 2).

O $\mathrm{PIC}^{10}$ utilizado é o da série $16 \mathrm{~F} 877^{11}$, que é programável e apresenta baixo custo ( U\$ 13,00). Possui internamente um conversor A/D de 10 bits, uma memória FLASH de $8 \mathrm{~Kb}$, uma EEPROM de 256 B e uma RAM de 368 B, 3 relógios ("timer"), 8 portas lógicas que podem ser configuradas para aquisição de si-

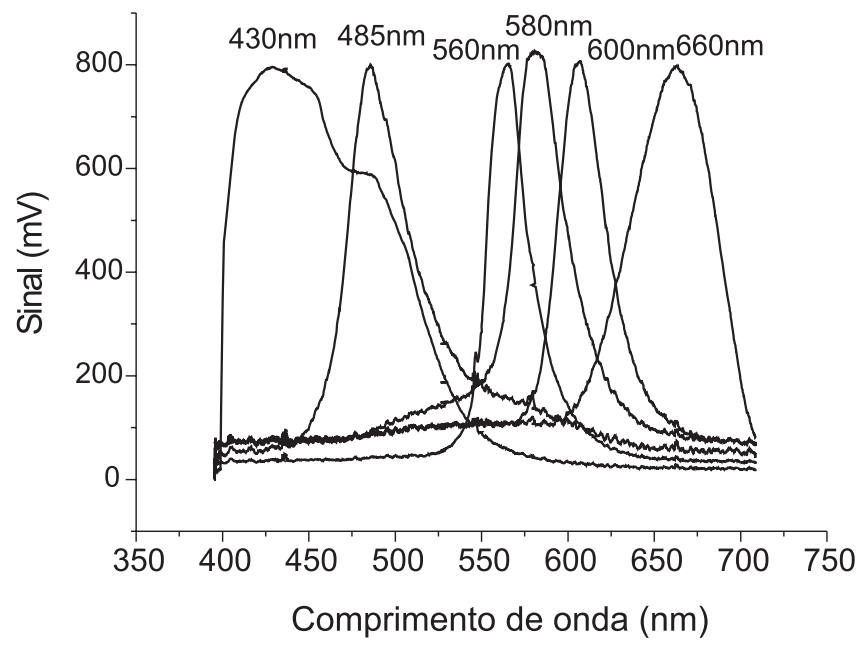

Figura 1. Espectros de emissão dos seis LEDs utilizados no fotômetro proposto 


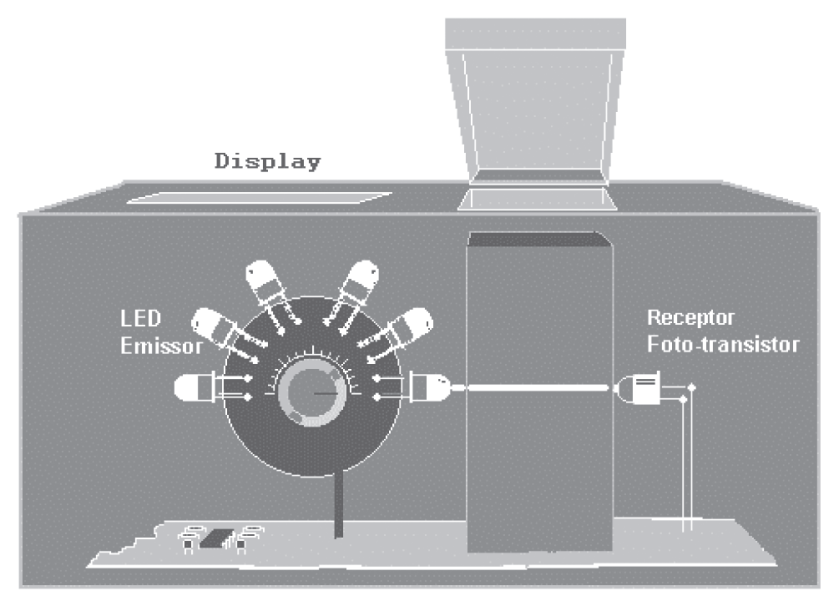

Figura 2. Diagrama esquemático do fotômetro proposto

nais, entre outros recursos importantes ${ }^{11}$. Esse PIC permite a introdução de filtros digitais e de periféricos de entrada e saída de dados (teclados, chaves, impressoras, mostradores, etc), além da redução drástica de componentes eletrônicos. Além disso, possibilita a introdução ou alteração de funcionalidades do instrumento sem necessidade de reconfiguração do hardware, bem como a realização de aquisição e tratamento de dados sem necessidade de microcomputador.

Um circuito eletrônico (Figura 3) foi projetado de modo a fornecer uma corrente mínima para funcionamento dos LEDs, o que garante uma boa estabilidade no sinal. Uma fonte de -5 e $+5 \mathrm{~V}$ foi montada para a alimentação desse circuito. Os sinais analógicos produzidos durante as medidas são enviados para o PIC, que os digitaliza por meio de um conversor A/D interno de 10 bits. Estes sinais são apresentados em valores de transmitância e absorbância em um mostrador de cristal líquido ("liquid crystal display-LCD") após conversão realizada por um software (escrito em linguagem C) gravado no microcontrolador. O código-fonte desse programa encontra-se disponível na homepage ${ }^{13}$ do nosso laboratório. Os sinais analógicos são também disponibilizados em uma saída lateral do fotômetro, para possibilitar seu monitoramento por um microcomputador mediante uma interface conversora (A/D).

O LCD utilizado no fotômetro é o modelo WH-1602A, o qual possui barramento de dados de 8 bits (podendo-se trabalhar com quatro ou oito bits) e barramento de controle de 3 bits. Neste trabalho, a comunicação entre o PIC e o mostrador foi sempre realizada empregando-se sete linhas, sendo três de controle e quatro para transmissão de dados de 4 bits.

Uma vez que o propósito principal deste trabalho foi desenvolver e construir um fotômetro multi-LED microcontrolado, o equipamento foi dotado de um periférico de saída (mostrador) dispensando assim o uso de um canal de saída de sinais. Todavia, um canal de saída analógica foi introduzido para que o usuário, se desejar, tenha acesso direto aos sinais que não foram ainda digitalizados. Apesar desses sinais poderem ser digitalizados por qualquer interface de comunicação, uma alternativa economicamente viável é usar a interface que foi desenvolvida pelo nosso grupo ${ }^{14}$. Essa interface permite a aquisição de sinais por meio da porta paralela do microcomputador.

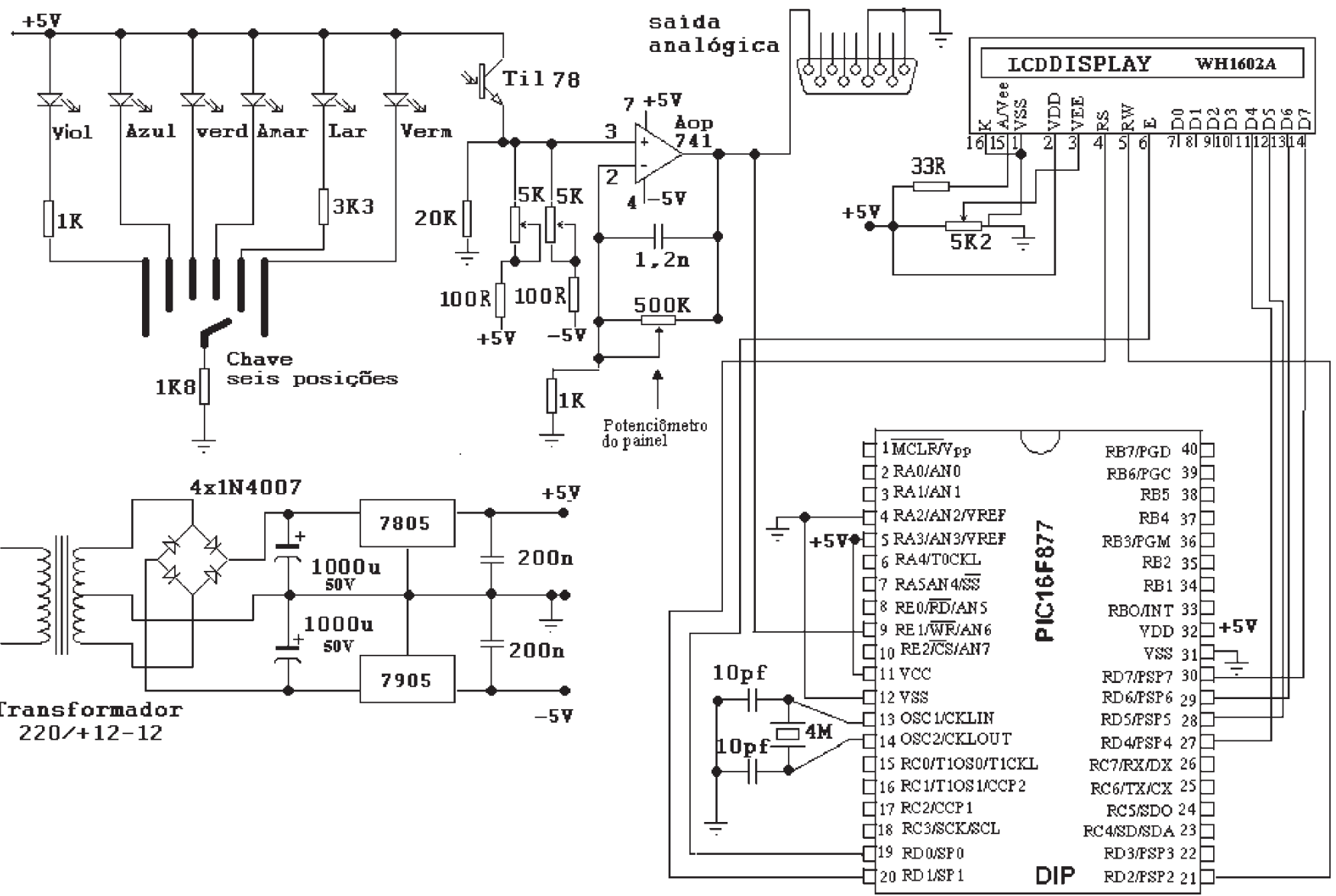

Figura 3. Esquema do circuito eletrônico do fotômetro desenvolvido 
Outra alternativa para transmissão dos sinais consiste na incorporação de um CI MAX232 $2^{15}$ no circuito eletrônico do fotômetro. Esse componente permite a transferência dos dados digitais, disponibilizados na saída RS do PIC, para a porta serial do microcomputador.

\section{Funcionamento do fotômetro e medidas analíticas}

O princípio de funcionamento do fotômetro proposto é muito simples: a radiação atravessa a cela de detecção contendo a amostra absorvente e alcança o fototransistor. As variações dos sinais detectados pelo circuito eletrônico são filtradas por um filtro passa-baixa. Em seguida, os sinais são amplificados por um pequeno circuito amplificador, conforme esquema mostrado na Figura 3.

Para realizar as medidas com o instrumento proposto, posicionase o LED correspondente ao comprimento de onda desejado e enche-se a cela de detecção com a solução do branco. O sinal de $100 \%$ em transmitância (correspondente a uma tensão de cerca de $4,0 \mathrm{~V}$ ) é ajustado pelo analista, girando-se outro botão existente no painel principal do fotômetro (Figura 2). Esse sinal é exibido no mostrador como o de máxima transmitância, sendo armazenado para posterior uso no cálculo das absorbâncias das soluções. Em seguida, o analista deve encher a cela com a solução absorvente (padrão ou amostra) e colocá-la no aparelho. Os valores das medidas de transmitância e absorbância são apresentados no mostrador LCD do fotômetro.

\section{Aplicações do fotômetro proposto}

\section{Análises de xaropes ferrosos}

Uma solução-estoque com $1000 \mathrm{mg} \mathrm{L}^{-1} \mathrm{em} \mathrm{Fe}^{2+}$ foi preparada a partir de $\mathrm{FeSO}_{4} .7 \mathrm{H}_{2} \mathrm{O}$ dissolvido em meio ácido $\left(\mathrm{H}_{2} \mathrm{SO}_{4}, 0,2\right.$ mol L $\left.{ }^{-1}\right)$. Soluções-padrão de $\mathrm{Fe}^{2+}\left(2,0 ; 4,0 ; 6,0 ; 8,0\right.$ e $\left.10,0 \mathrm{mg} \mathrm{L}^{-1}\right)$ foram preparadas a partir da solução-estoque, por diluição com água deionizada. Essas soluções foram utilizadas na construção da curva analítica do fotômetro proposto para determinação de $\mathrm{Fe}^{2+}$, baseada em medidas de absorbância usando o método colorimétrico 1,10-fenantrolina ${ }^{16}$. As amostras de xarope ferroso foram adquiridas em drogarias locais e analisadas após diluição com água deionizada.

\section{Análises bioquímicas clínicas}

Os reagentes e as soluções-padrão utilizadas nas determinações bioquímicas clínicas foram adquiridos de kits comerciais ${ }^{16-23}$. A preparação dos reagentes foi realizada de acordo com procedimento descrito no manual de instruções do kit correspondente a cada parâmetro bioquímico. A escolha do LED apropriado para cada determinação também foi norteada pelas informações disponíveis nesses manuais. Com efeito, o LED verde do fotômetro proposto foi usado para determinação de proteínas totais ${ }^{17}$ (baseada no método do biureto ${ }^{18}$ ) e de glicose $^{19}$, o LED vermelho para albumina $^{20}$, o amarelo para cálcio ${ }^{21}$, o laranja para uréia ${ }^{22}$, o azul

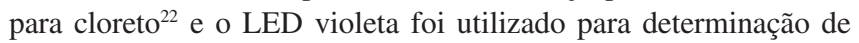
hemoglobina ${ }^{23}$. As amostras de soro sangüíneo foram fornecidas pelo Laboratório de Análises Clínicas do Hospital Universitário da Universidade Federal da Paraíba e analisadas sem nenhum tratamento prévio.

As amostras de xarope ferroso foram também analisadas usando dois espectrofotômetros de absorção UV-VIS comerciais (Hewlett Packard-HP, modelo 8453 e outro da Micronal, modelo B342II), enquanto que para as amostras de soro sangüíneo utilizou-se apenas o instrumento da HP.

\section{RESULTADOS E DISCUSSÃO}

Não obstante a simplicidade da eletrônica utilizada no fotômetro desenvolvido, o que permitiu diminuir consideravelmente seu custo total, seu desempenho não foi comprometido. De fato, sua estabilidade foi investigada por um período de $8 \mathrm{~h}$ de funcionamento e nenhuma variação considerável do sinal produzido foi observada.

O desempenho do fotômetro proposto foi inicialmente avaliado mediante a determinação de íon ferroso em amostras de xarope. Os resultados são apresentados na Tabela 1 , juntamente com os obtidos com os instrumentos HP e Micronal. Uma boa concordância entre os valores obtidos empregando os três equipamentos pode ser observada. De fato, nenhuma diferença significativa, ao nível de $95 \%$ de probabilidade, foi verificada entre os resultados quando o teste $t$ emparelhado foi aplicado. O fotômetro proposto também forneceu resultados com precisão satisfatória (Tabela 1), sendo atestada por um desvio padrão relativo (RSD) geralmente menor ou igual a $0,3 \%$ $(n=5)$, enquanto os valores de RSD obtidos com os instrumentos comerciais foram usualmente menores que $0,2 \%(n=5)$.

Tabela 1. Resultados das determinações de íon ferroso em xaropes usando o fotômetro proposto e dois instrumentos comerciais

\begin{tabular}{ccrr}
\hline Amostra & \multicolumn{3}{c}{$\left[\mathrm{Fe}^{2+}\right] \times 10^{3}\left(\mathrm{mg} \mathrm{L}^{-1}\right)$} \\
& Fotômetro proposto & \multicolumn{1}{c}{ Micronal } & \multicolumn{1}{c}{ HP } \\
\hline 1 & $124,7( \pm 0,3)$ & $124,5( \pm 0,1)$ & $124,1( \pm 0,1)$ \\
2 & $129,0( \pm 0,3)$ & $129,0( \pm 0,1)$ & $128,7( \pm 0,2)$ \\
3 & $24,9( \pm 0,5)$ & $25,0( \pm 0,1)$ & $25,0( \pm 0,1)$ \\
4 & $124,9( \pm 0,2)$ & $124,7( \pm 0,1)$ & $125,0( \pm 0,1)$ \\
5 & $25,2( \pm 0,3)$ & $25,3( \pm 0,1)$ & $25,3( \pm 0,1)$ \\
6 & $123,0( \pm 0,2)$ & $123,3( \pm 0,1)$ & $123,7( \pm 0,1)$ \\
7 & $123,8( \pm 0,2)$ & $123,0( \pm 0,1)$ & $123,3( \pm 0,0)$ \\
\hline
\end{tabular}

* Valores entre parênteses são as estimativas dos desvios padrões relativos para 5 determinações repetidas.

Para a determinação de $\mathrm{Fe}^{2+}$, as medidas de absorbância foram realizadas usando o LED azul, pois este apresenta, entre os LEDs disponíveis, o pico de emissão $\left(\lambda_{\text {máx }}=485 \mathrm{~nm}\right)$ mais próximo do máximo de absorção do complexo Fe(II)-fenantrolina $\left(\lambda_{\text {máx }} \approx 510\right.$ $\mathrm{nm})$. Apesar de não existir coincidência total entre ambos os máximos, não houve problemas de desvios da lei de Beer em virtude da banda de emissão do LED azul ser relativamente estreita (Figura 1). A Tabela 2 mostra que o fotômetro proposto apresentou uma linearidade na curva analítica tão boa quanto à da curva obtida com os espectrofotômetros comerciais. Com efeito, um coeficiente de correlação maior que 0,999 é considerado como evidência de um ajuste ideal dos dados para a reta de regressão ${ }^{24}$. Contudo, é provável que ocorresse algum problema de desvio da linearidade se as medidas de absorbância fossem realizadas, para esta aplicação, empregando o LED violeta, cuja banda de emissão é consideravelmente mais larga (Figura 1). Quanto ao fototransistor, este normalmente não apresenta problemas de linearidade em sua res-

Tabela 2 - Outras características ${ }^{24}$ usadas para comparação do desempenho do fotômetro proposto em relação aos instrumentos comerciais utilizados nas determinações de $\mathrm{Fe}^{2+}$

\begin{tabular}{lccc}
\hline Características & Fotômetro proposto & Micronal & HP \\
\hline Linearidade* & 0,99960 & 0,99978 & 0,99954 \\
LD** $\left(\mathrm{mg} \mathrm{L}^{-1}\right)$ & $1,9 \times 10^{-2}$ & $1,2 \times 10^{-2}$ & $3,3 \times 10^{-4}$ \\
LQ $^{* *}\left(\mathrm{mg} \mathrm{L}^{-1}\right)$ & $5,7 \times 10^{-2}$ & $3,6 \times 10^{-2}$ & $1,0 \times 10^{-3}$ \\
\hline
\end{tabular}

* Expressa em termos do coeficiente de correlação, $\mathbf{r}(\mathrm{n}=5) ; * * \mathrm{LD}=$ limite de detecção e LQ = limite de quantificação 
Tabela 3. Resultados das determinações de parâmetros bioquímicos clínicos usando o instrumento proposto e um espectrofotômetro HP

\begin{tabular}{|c|c|c|c|c|c|c|c|c|}
\hline \multirow[t]{2}{*}{ Parâmetro } & \multicolumn{2}{|c|}{ Amostra 1} & \multicolumn{2}{|c|}{ Amostra 2} & \multicolumn{2}{|c|}{ Amostra 3} & \multicolumn{2}{|c|}{ Amostra 4} \\
\hline & LED & HP & LED & HP & LED & HP & LED & $\mathrm{HP}$ \\
\hline Proteínas Totais $\left(\mathrm{g} \mathrm{dL}^{-1}\right)$ & 3,8 & 3,8 & 4,6 & 4,5 & 4,9 & 4,9 & 4,2 & 4,2 \\
\hline Albumina $\left(\mathrm{g} \mathrm{dL}^{-1}\right)$ & 3,9 & 3,9 & 3,4 & 3,2 & 3,3 & 3,4 & 4,4 & 4,4 \\
\hline Glicose $\left(\mathrm{mg} \mathrm{dL}^{-1}\right)$ & 186,0 & 192,0 & 73,9 & 73,2 & 96,0 & 90,3 & 109,8 & 110,3 \\
\hline Cálcio $\left(\mathrm{mg} \mathrm{dL}^{-1}\right)$ & 7,2 & 7,8 & 8,8 & 9,1 & 8,8 & 9,2 & 8,7 & 8,7 \\
\hline Uréia $\left(\mathrm{mg} \mathrm{dL}^{-1}\right)$ & 29 & 31 & 15 & 14 & 27 & 27 & 15 & 15 \\
\hline Cloreto $\left(\mathrm{mEq} \mathrm{L}{ }^{-1}\right)$ & 88,1 & 87,0 & 83,9 & 82,9 & 93,5 & 92,2 & 93,8 & 94,0 \\
\hline Hemoglobina $\left(\mathrm{mg} \mathrm{dL}^{-1}\right)$ & 14,0 & 14,5 & 15,5 & 14,5 & 13,5 & 14,0 & 12,6 & 11,7 \\
\hline
\end{tabular}

posta, em virtude de sua função de transferência apresentar uma relação linear entre a potência da radiação incidente e o sinal elétrico produzido ${ }^{25}$.

Os limites de detecção e quantificação do fotômetro proposto e dos instrumentos comerciais foram estimados, em nível de $95 \%$ de confiança, empregando o método baseado em parâmetros da curva analítica e no desvio padrão do branco ${ }^{24}$. Os resultados são apresentados na Tabela 2, os quais mostram uma diferença pequena entre os valores obtidos com o fotômetro proposto e o Micronal. Por outro lado, o espectrofotômetro HP ofereceu limites de detecção e quantificação melhores que os outros dois instrumentos. Apesar disso, o desempenho do fotômetro desenvolvido pode ser também considerado satisfatório quando avaliado em termos dessa figura de mérito.

O desempenho do fotômetro proposto foi também avaliado mediante a realização de análises bioquímicas clínicas e os resultados são mostrados na Tabela 3. Neste caso, apenas o espectrofotômetro HP foi utilizado para fins de comparação entre os desempenhos analíticos. Todavia, nesta aplicação não foram construídas curvas analíticas, pois os métodos colorimétricos descritos nos manuais dos kits baseiam-se sempre em um único padrão que é disponibilizado para cada espécie bioquímica. Os valores encontrados na Tabela 3 representam sempre a média de três determinações analíticas repetidas $(n=3)$. Novamente, é notável a boa concordância entre os valores obtidos com o fotômetro proposto e o instrumento comercial HP. Com efeito, nenhuma diferença significativa, em nível de $95 \%$ de confiança, foi verificada entre os resultados quando o teste $t$ emparelhado foi aplicado. Por outro lado, os valores de RSD obtidos nessas determinações foram usualmente menores que $0,5 \%$ para ambos os instrumentos.

\section{CONCLUSÕES}

O fotômetro proposto apresentou um desempenho bastante satisfatório como demonstrado nas aplicações analíticas realizadas, as quais permitiram atestar sua eficiência, versatilidade e flexibilidade. De fato, foi possível determinar satisfatoriamente o íon ferroso em amostras de xarope, bem como sete parâmetros bioquímicos clínicos, a partir de medidas realizadas usando seis diferentes LEDs, simplesmente girando-se um pequeno botão existente no painel do equipamento. A determinação desses parâmetros foi motivada não apenas pela sua importância no diagnóstico de certos estados patológicos do paciente, mas também - e principalmente - por permitirem o uso de todos os LEDs disponíveis no fotômetro. Neste sentido, o instrumento proposto demonstrou ter potencial para realização de análises multicomponentes.

$\mathrm{O}$ custo do fotômetro multi-LED foi estimado como U\$30.00, o qual é bem inferior ao de qualquer instrumento comercial simples, cujo preço é de cerca de U\$ 1,500.00. O fotômetro microcontrolado proposto também oferece manutenção simples e barata, pois utiliza uma eletrônica simples, componentes baratos e facilmente encontrados no comércio.

As características destacadas fazem do fotômetro proposto uma ferramenta alternativa economicamente viável para análises químicas em laboratórios de rotina, de pesquisa e/ou de ensino carentes de recursos financeiros. Finalmente, por ser microcontrolado e portátil, dispensa o uso de microcomputador e também possibilita a realização de análises químicas de campo.

\section{AGRADECIMENTOS}

Ao CNPq e à CAPES pelas bolsas concedidas.

\section{REFERÊNCIAS}

1. Dasgupta, P. K.; Bellamy, H. S.; Liu, H.;Lopes, J. L.; Loree, E. L.; Morris, K. J.; Petersen, K.; Mir, K. A.; Talanta 1993, 40, 53.

2. Dasgupta, P. K.; Eom, I. Y.; Morris, K. J.; Li, J.; Anal. Chim. Acta 2003, 500, 337.

3. Gaião, E. N.; Honorato, R. S.; Santos, S. R. B.; Araújo, M. C. U.; Analyst 1999, 124, 1727.

4. Pasquini, C; Raimundo Jr., I. M.; Quim. Nova 1984, 7, 24.

5. Araujo, M. C. U.; Santos, S. R. B.; Silva, E. A.; Veras, G.; Lima, J. L. F. C; Lapa, R. A. S.; Quim. Nova 1997, 20, 137.

6. Pereira, E. A.; Cardoso, A. A.; Dasgupta, P. K.; Quim. Nova 2001, 24, 443.

7. Rocha, F. R. P.; Martelli, P. B.; Reis, B. F.; J. Braz. Chem. Soc. 2004, 15, 38.

8. Fonseca, A.; Raimundo Jr., I. M.; Anal. Chim. Acta 2004, 522, 223.

9. Cantrell, M. K.; Ingle, J. D.; Anal. Chem. 2003, 75, 27.

10. Pereira, F.; PIC, Programação em C, 2eㅡ. érica: São Paulo, 2003.

11. http://www.microchip.com, acessada em Outubro 2004.

12. http://www.winstar.com.tw/, acessada em Janeiro 2005.

13. http://www.laqa.quimica.ufpb.br.

14. Gaião, E. N.; Medeiros, E. P.; Lyra, W. S.; Moreira, P. N. T.; Silva, E. C.; Araújo, M. C. U.; Quim. Nova 2004, 27, 825.

15. http://focus.ti.com/lit/ds/symlink/max232.pdf, acessada em Outubro 2004.

16. ASTM E 394-70; Standard test method for iron in trace quantities using the 1,10-phenanthroline method.

17. QUIBASA - QUÍMICA BÁSICA; Bioclin: Método Colorimétrico para Proteínas Totais e Albumina, Belo Horizonte: QUIBASA, 2003.

18. Gornall, A. G.; Bardawill, C. S.; David, M. M.; J. Biol. Chem. 1949, 177, 751.

19. DOLES; Sistema Colorimétrico para Quantificação da Glicose no Soro e Líquidos Biológicos, Goiânia, 2003.

20. DOLES; Sistema Colorimétrico para Quantificação da Albumina no Soro e Líquidos Biológicos, Goiânia, 2003.

21. LABTEST DIAGNÓSTICA; Sistemas para Diagnósticos: Cálcio liquiform, Lagoa Santa, 2003.

22. LABTEST DIAGNÓSTICA; Sistemas para Diagnósticos: Uréia ce, Lagoa Santa, 2003.

23. LABTEST DIAGNÓSTICA; Sistemas para Diagnósticos: Cloreto, Lagoa Santa, 2003.

24. Ribani, M.; Bottoli, C. B. G.; Collins, C. H.; Jardim, I. C. S. F.; Melo, L. F. C.; Quim. Nova 2004, 27, 771.

25. http://sharp-world.com/products/device/ctlg/esite23/table/072.html, acessada em Outubro 2004. 